\title{
Relation entre dilution de l'azote et cinétique de croissance chez les graminées
}

\author{
Michel CALOIN \& Olivier YU \\ Institut de Botanique, Laboratoire d'Ecologie végétale, 28, rue Goethe, F 67083 Strasbourg Cedex
}

RÉSUMÉ

\begin{abstract}
La relation entre la production de biomasse et la teneur en azote de la plante en phase végétative est étudiée chez des individus isolés de Dactylis glomerata L. cultivés en milieu hydroponique et dans des conditions d'environnement contrôlées. Les différents traitements expérimentaux incluant l'effet de la concentration en nitrate, de l'éclairement et de la température, confirment la généralité du processus de dilution de l'azote dans la plante au cours de la croissance.

Ce processus est analysé sur la base d'un modèle de croissance à deux composantes. La variation de la composition chimique de la plante est décrite en termes de variation du rapport de ces composantes. Dans cette approche, la teneur en azote de la plante varie linéairement avec la vitesse de croissance relative, en accord avec nos observations expérimentales. Nous montrons que cette relation permet de rendre compte de la variation de la teneur en azote au cours de la croissance, mesurée dans le cas de cultures de différentes graminées en milieu naturel.
\end{abstract}

Mots clés additionnels : Dactylis glomerata L., phase végétative, teneur en azote, modèle de croissance.

The relationship between plant nitrogen content and biomass production during the vegetative phase was studied in individual plants of Dactylis glomerata L., grown hydroponically in a controlled environment. The experiments, performed under different conditions of nitrate concentration, light intensity and temperature, confirmed the dilution of nitrogen in the plant during growth. This process of dilution was analysed on the basis of a two-componend model. The variation in chemical composition of a plant was described in terms of the variation of the ratio of these components. In this approach, nitrogen content varied linearly with relative growth rate. It was shown that this relationship could provide a framework for the description of change in nitrogen content during growth of various Gramineae in a natural environment.

Additional key words : Dactylis glomerata L., vegetative phase, nitrogen content, growth model.

\section{INTRODUCTION}

L'analyse de la variation de la teneur en azote de graminées en phase végétative conduit à distinguer au moins deux phases dans le processus de croissance (HELler, 1969 ; GiLlet, 1980). Dans les jours qui suivent la germination ou le début de la repousse après chaque coupe, on observe une accélération de la production de biomasse ; parallèlement, la teneur en azote de la matière sèche augmente, passant de 2 à 5 p. 100 environ dans le cas du dactyle. On note alors un ralentissement de la croissance relative qui s'accompagne d'une diminution de la teneur en azote. Ce processus se poursuit sur toute la durée de la phase végétative. Il en résulte une variation des besoins en azote de la plante au cours de la croissance, qui doit être prise en compte si l'on désire optimiser l'apport des engrais azotés (FrISSEL \& VAN VEEN, 1981; GREENWOOD, 1981 ; GILLET et al., 1984).

Le processus de dilution de l'azote dans la matière sèche au cours de la croissance a été principalement décrit et discuté dans le cas de cultures de graminées en milieu naturel (LĖMAIRE \& SALETTE, 1984). Dans ces expériences, il est cependant difficile d'assurer que le niveau de nutrition azotée n'est pas limitant en cours de croissance, ce qui pourrait expliquer au moins partiellement l'allure des courbes de dilution de l'azote. Dans la présente étude, nous avons tenté de contrôler la généralité de ce processus en caractérisant la croissance du dactyle cultivé en conditions hydroponiques et en environnement contrôlé. Nous avons en particulier étudié l'effet de la concentration en nitrate du milieu de culture. L'analyse et la comparai- 
son des différents traitements expérimentaux nous a amenés à proposer, sur la base d'un modèle cinétique, une relation entre la teneur en azote de la plante et la vitesse de croissance relative (CALOIN \& YU, 1984). Nous montrons que cette description permet de rendre compte, dans le cas de culture en milieu naturel, des relations entre la teneur en azote de la partie aérienne et la production de biomasse proposées par MIYASAKA et al. (1975) pour le riz et par SALETTE \& LEMAIRE (1981) pour différentes graminées prairiales.

\section{MATÉRIEL ET MÉTHODES}

Les plantes de Dactylis glomerata L., cv. «Floréal ", sont cultivées en conditions hydroponiques dans des bacs de 351 , à raison de 32 pots par bac. Les expériences, limitées à la phase végétative, sont réalisées dans des chambres climatisées, aux températures de 10 à $25^{\circ} \mathrm{C}$. Les conditions de culture sont les suivantes :

- concentration en nitrate du milieu nutritif : 0,5 et $20 \mathrm{mM}$;

- éclairement : à $25^{\circ} \mathrm{C}, 3$ niveaux : 35,55 et $85 \mathrm{~W} . \mathrm{m}^{-2}$; à $10^{\circ} \mathrm{C}, 55 \mathrm{~W} . \mathrm{m}^{-2}$ seulement.

Le milieu nutritif que nous utilisons est celui décrit par BLONDON (1964) qui a été ajusté aux 2 teneurs en nitrate étudiées selon le principe suivant : les principaux anions autres que $\mathrm{NO}_{3}^{-}$(sulfates et phosphates) sont constants. Les cations $\mathrm{K}^{+}, \mathrm{Ca}^{2+}$ et $\mathrm{Mg}^{2+}$ varient, mais leur rapport reste constant (COİC et al., 1974). La concentration des oligoélements est identique pour les 2 milieux. La composition des solutions nutritives est donnée en annexe.

La concentration en nitrate du milieu nutritif est régulièrement mesurée à l'aide d'une électrode spécifique et les solutions sont renouvelées jusqu'à 3 fois par semaine pour que la concentration en nitrate soit toujours supérieure à 80 p. 100 de sa teneur initiale. Les solutions sont aérées pendant $10 \mathrm{mn}$ toutes les $2 \mathrm{~h}$.

L'éclairement $(16 \mathrm{~h} / \mathrm{j})$ provient de tubes fluorescents OSRAM L $65 \mathrm{~W} / 19(400-700 \mathrm{~nm})$ et le rayonnement global reçu au niveau des plantes est contrôlé chaque semaine au moyen d'une pile de Moll.

En début d'expérience, nous avons homogénéisé les plantes issues des semis en ne retenant que les individus présentant 3 feuilles apparentes sur la talle principale. Au $1^{\text {er }}$ prélèvement, pour l'ensemble des traitements, les plantes ont entre 4 et 5 feuilles. A la fin des expériences, qui ont duré 3 à 5 mois selon la condition des températures $\left(25\right.$ ou $\left.10^{\circ} \mathrm{C}\right)$, ce nombre de feuilles est de l'ordre de 14. Nous avons effectué environ 10 prélèvements par traitement. A chaque prélèvement, de façon à tenir compte de l'hétérogénéité dans le développement, les plantes sont classées selon leur nombre de feuilles sur la talle principale. Un échantillon de 8 à 15 plantes par traitement est alors prélevé proportionnellement au nombre d'individus dans chacune des classes. Au fur et à mesure des prélèvements, le nombre de plantes par bac diminue. Les plantes sont alors redistribuées dans chacun des bacs de façon à éviter un recouvrement trop important des parties aériennes. Ce recouvrement n'est intervenu que pour des valeurs de poids sec supérieures à $1 \mathrm{~g}$, mais par suite du protocole suivi, il est toujours resté relativement faible, même en fin d'expérience.

Le poids sec des parties aérienne et racinaire des plantes individuelles est mesuré après séchage durant au moins $3 \mathrm{j}$ à $70^{\circ} \mathrm{C}$. Les teneurs en azote sont mesurées séparément pour les parties aérienne et racinaire par la méthode de KJELDAHL (dosage de l'azote organique et d'une partie de l'azote nitrique) avec une erreur relative inférieure à 3 p. 100 .

\section{RÉSULTATS EXPÉRIMENTAUX}

\section{A. Cinétiques de croissance expérimentales}

L'effet des conditions du milieu de culture sur la croissance de $D$. glomerata est illustré par la figure 1 en représentation semi-logarithmique. Ainsi que nous l'avons précédemment décrit, chaque valeur de poids sec $M(t)$ portée sur ce graphique a été déterminée à partir d'un échantillonnage de 8 à 15 plantes ; la précision relative sur chacune des valeurs, de l'ordre de 5

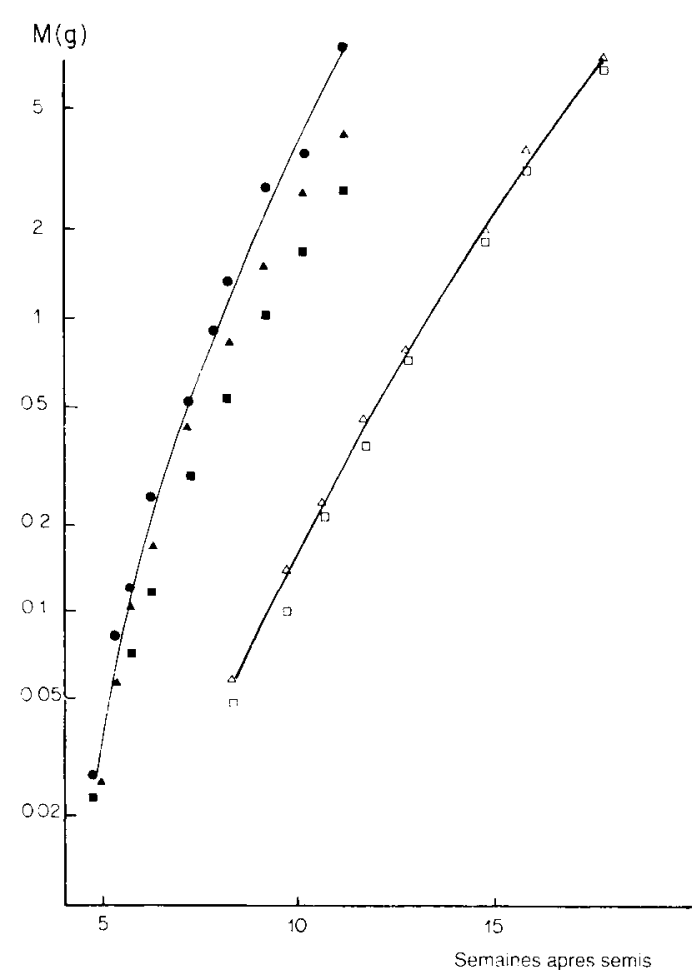

Figure 1

Poids sec par plante $M$ en fonction du temps.

A $10{ }^{\circ} \mathrm{C}$, éclairement : $55 \mathrm{~W} \cdot \mathrm{m}^{-2}$. Concentration en nitrate $d u$ milieu nutritif : $\square 0,5 \mathrm{mM} ; \triangle 20 \mathrm{mM}$.

A $25{ }^{\circ} \mathrm{C}$, éclairement: - $35 \mathrm{~W} \cdot \mathrm{m}^{-2}$; $\mathbf{\Delta} 55 \mathrm{~W} \cdot \mathrm{m}^{-2}$; - $85 \mathrm{~W} . \mathrm{m}^{-2}$. Concentration en nitrate du milieu nutritif : $20 \mathrm{mM}$. Les courbes en trait continu illustrent les variations expérimentales aux deux conditions de température.

Dry weight, $M$, per plant with time.

At $10{ }^{\circ} \mathrm{C}$, irradiance : $55 \mathrm{~W} \cdot \mathrm{m}^{-2}$. Concentration of nitrate in the nutrient solution : $\square$ $0.5 \mathrm{mM} ; \triangle 20 \mathrm{mM}$.

At $25{ }^{\circ} \mathrm{C}$, irradiance: $35{\mathrm{~W} . \mathrm{m}^{2}}^{2}$; $155 \mathrm{~W}, \mathrm{~m}^{-2}$; - $85 \mathrm{~W} . \mathrm{m}^{-2}$. Concentration of nitrate in the nutrient solution : $20 \mathrm{mM}$.

The continuous lines are only visual guides. 
à 10 p. 100 , est approximativement constante sur toute la durée de l'expérience. En accord avec les observations antérieures (CHAMPAGNAT et al., 1969 ; EVANS, 1972), la croissance est plus rapide à $25^{\circ} \mathrm{C}$ qu'à $10^{\circ} \mathrm{C}$, ainsi qu'aux plus forts éclairements. En revanche, l'effet de la concentration en nitrate du milieu nutritif est peu apparent. Ce résultat s'explique au moins partiellement par le fait que les concentrations en nitrate utilisées sont relativement élevées (CLEMENT et al., 1978) et que par suite du renouvellement fréquent du milieu nutritif, la concentration en nitrate est maintenue aussi constante que possible.

Les courbes de croissance de la figure 1 présentent une $1^{\text {re }}$ phase linéaire, correspondant à une croissance exponentielle, suivie d'une phase de ralentissement. Cette phase de ralentissement est plus marquée à $25^{\circ} \mathrm{C}$ qu'à $10^{\circ} \mathrm{C}$, température pour laquelle la croissance est approximativement exponentielle sur toute la durée de l'expérience.

Ces comportements cinétiques peuvent être caractérisés quantitativement par la vitesse de croissance relative définie par

$$
r(t)=\frac{1}{M} \frac{d M}{d t}
$$

qui correspond à la pente des courbes de croissance dans la représentation semi-logarithmique. Dans le cas d'une croissance exponentielle, $r(t)$ est constant. Les valeurs de $r(t)$ ont été déterminées graphiquement pour chacune des mesures de poids sec. L'estimation graphique de la pente étant imprécise en début et en fin d'expérience, nous n'avons pas calculé les valeurs de $r(t)$ pour les 2 valeurs extrêmes de la biomasse. Les figures $2 \mathrm{~A}$ et $2 \mathrm{~B}$ illustrent respectivement la variation de la vitesse de croissance relative en fonction du temps et en fonction du poids sec des plantes individuelles. Pour une même valeur de poids sec (fig. 2B), la vitesse de croissance relative est plus élevée à $25^{\circ} \mathrm{C}$ qu'à $10^{\circ} \mathrm{C}$, mais cette différence s'atténue au cours de la croissance. On peut également remarquer que la vitesse de croissance relative diminue plus fortement en début de croissance, quand il n'y a pas encore de recouvrement des parties aériennes; par la suite, elle ne présente plus qu'une faible variation. On peut donc penser que, dans nos conditions expérimentales, les cinétiques que nous avons mesurées sont représentatives de la croissance d'individus isolés.

\section{B. Variation de la teneur en azote au cours de la croissance}

Les figures $3 \mathrm{~A}$ et $3 \mathrm{~B}$ montrent les variations de la teneur en azote $\mathrm{n}$ en fonction du temps et en fonction du poids sec des plantes individuelles. Les variations de $\mathrm{n}$ sont très différentes aux 2 températures. En début de croissance, les teneurs en azote sont approximativement les mêmes à 10 et $25^{\circ} \mathrm{C}$; mais la diminution de la teneur en azote est beaucoup plus accentuée à $25^{\circ} \mathrm{C}$. Ce résultat est particulièrement apparent sur la figure $3 \mathrm{~B}$ qui compare les teneurs en azote des plantes pour une même valeur de poids sec. On observe en revanche peu d'effet lié à la concentration en nitrate du milieu nutritif et à l'éclairement.

Pour l'ensemble des traitements, la teneur en azote des racines (2 à 2,5 p. 100) reste approximativement
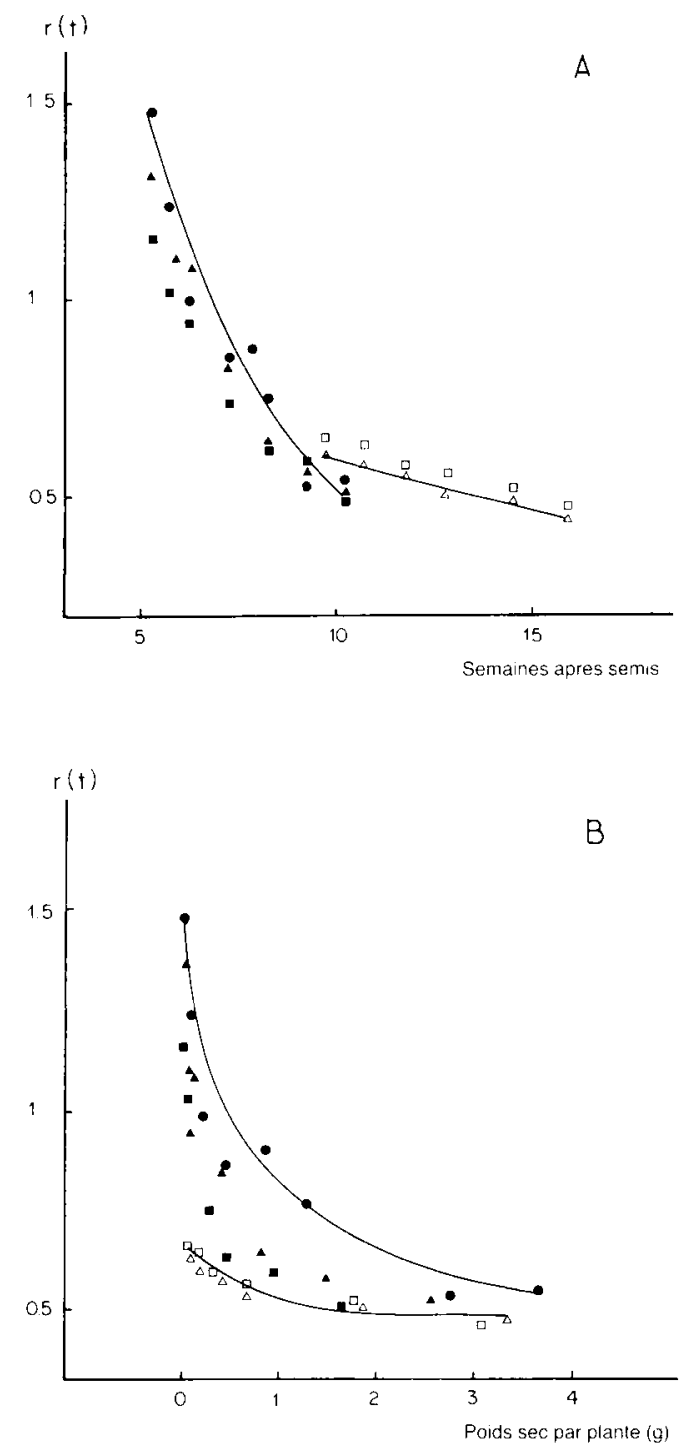

Figure 2

Vitesse de croissance relative $r(t)$ en fonction du temps $(A)$ et en fonction du poids sec des plantes individuelles $(B)$. Mêmes conventions que pour la figure 1.

Relative growth rate, $r(t)$, with $(A)$, time and $(B)$, dry weight of individual plants. Same conventions as in figure $l$.

constante. La variation de $\mathrm{n}$ reflète donc essentiellement la variation de la teneur en azote de la partie aérienne. On peut également préciser que, pour l'ensemble des traitements, les parties racinaires représentent 30 p. 100 du poids sec des plantes en début de croissance. Ce pourcentage décroît progressivement jusqu'à une valeur voisine de 15 en fin d'expérience. La température joue donc un rôle important dans la variation de la teneur en azote au cours de la croissance, mais modifie peu la répartition des assimilats entre les parties aérienne et racinaire.

La comparaison des figures 2 et 3 fait apparaître une analogie dans les variations de $r$ et de $n$ au cours de la croissance. Dans le chapitre suivant, nous montrons qu'il est possible de relier ces variations sur la base d'un modèle de croissance à 2 composantes (CALOIN \& YU, 1982, 1984), et nous discutons également différents travaux antérieurs décrivant la variation de la teneur en azote au cours de la croissance dans le cas de cultures en milieu naturel. 

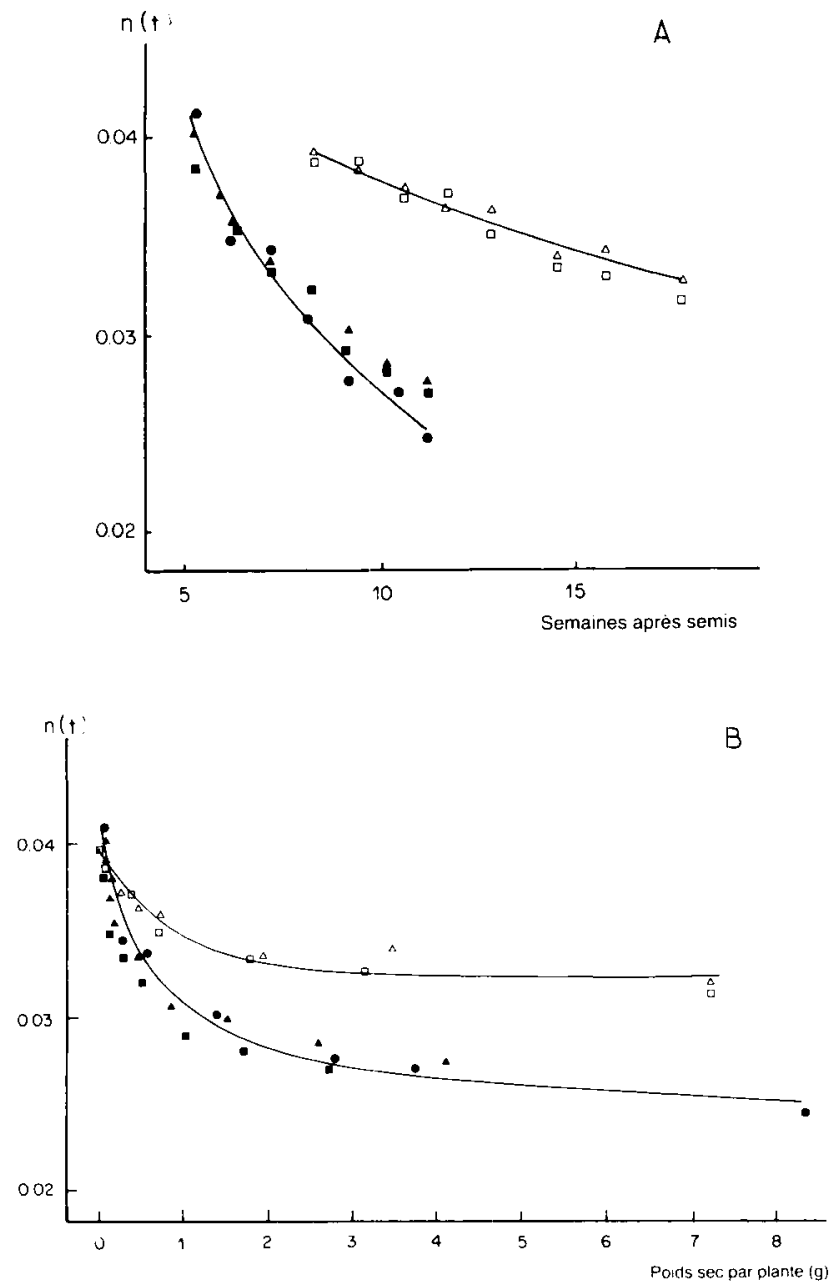

Figure 3

Teneur en azote $n(t)$ en fonction du temps $(A)$ et en fonction $d u$ poids sec des plantes individuelles (B). Mêmes symboles que pour la figure 1. Les courbes en trait continu de la figure $3 B$ sont calculées à partir de l'équation (16) pour le traitement correspondant à la même concentration en nitrate du milieu nutritif (20 mM).

Nitrogen content, $n(t)$, with (A), time and $(B), d r y$ weight of individual plants. Continuous lines in figure $3 B$ were calculated by equation (16) for the same condition of concentration of nitrate in the nutrient solution $(20 \mathrm{mM})$. Same symbols as in figure 1 .

\section{DISCUSSION}

\section{A. Modèle de croissance à 2 composantes}

Les 2 composantes du modèle sont définies en référence avec le modèle de croissance exponentiel qui s'exprime par l'équation

$$
\frac{\mathrm{dM}}{\mathrm{dt}}=\mathrm{k}_{0} \mathrm{M}(\mathrm{t})
$$

où $\mathrm{k}_{0}$ est une constante pour une condition d'environnement donnée. La biomasse produite à l'instant $t$ est proportionnelle à la biomasse totale de la plante. Sur la base de ce modèle, on peut faire l'hypothèse que le ralentissement observé dans le processus de croissance reflète l'existence d'une composante de la plante qui serait inactive dans les processus de production de biomasse. Dans cette description, la $1^{\text {re }}$ composante
$\mathbf{M}_{1}$, associée aux processus de production de biomasse (en particulier photosynthèse et nutrition minérale), est définie à partir de la relation

$$
\frac{\mathrm{dM}}{\mathrm{dt}}=\mathrm{k}_{1} \mathrm{M}_{1}(\mathrm{t})
$$

$\operatorname{avec} M_{1}(t) \leqslant M(t)$

où $k_{1}$ est une constante pour un environnement donné. On en déduit

$$
\mathrm{M}_{1}=\frac{1}{\mathrm{k}_{1}} \frac{\mathrm{dM}}{\mathrm{dt}} .
$$

La $2^{\mathrm{e}}$ composante,

$$
\mathbf{M}_{2}=\mathbf{M}-\mathbf{M}_{1} \text {, }
$$

n'intervient pas dans les processus de production de biomasse. Dans le cas de la croissance exponentielle, la plante ne comprend que la composante $\mathrm{M}_{1}$ $\left(\mathrm{M}_{1}=\mathrm{M}, \mathrm{M}_{2}=0\right)$.

A partir de la définition de ces composantes qui s'accorde avec des travaux antérieurs (en particulier WARREN WILSON, 1972; COOPER \& THORNLEY, 1976), il est possible d'évaluer le rapport $p_{1}(t)$ entre la composante $M_{1}$ et la biomasse totale $M$ en écrivant

$$
\mathrm{p}_{1}(\mathrm{t})=\frac{\mathrm{M}_{1}}{\mathrm{M}}=\frac{1}{\mathrm{Mk}_{1}} \frac{\mathrm{dM}}{\mathrm{dt}}=\frac{\mathrm{r}(\mathrm{t})}{\mathrm{k}_{1}} .
$$

Ce rapport est proportionnel à la vitesse de croissance relative et décroît au cours du temps. Si l'on admet qu'en début de croissance $M_{1}$ est peu différent de $M\left(p_{1} \simeq 1\right.$, croissance quasi-exponentielle), $k_{1}$ peut être estimé à partir de la pente initiale des courbes de croissance dans la représentation semi-logarithmique (fig. 1). Ces valeurs de $k_{1}$ sont portées dans le tableau 1. Il est alors possible, à l'aide de la relation (6), de calculer les variations de $p_{1}$ au cours de la croissance (fig. 4A et 4B). Ces variations montrent des différences importantes aux 2 conditions de température. En début d'expérience, ainsi que nous l'avons postulé, $\mathrm{p}_{1}$ est voisin de 1 . En fin d'expérience, pour des valeurs de poids secs de $2,5 \mathrm{~g}, \mathrm{p}_{1}$ est de l'ordre de 0,65 à $10^{\circ} \mathrm{C}$, alors qu'il est inférieur à 0,4 à $25^{\circ} \mathrm{C}$.

Par suite de leur fonction différente, les 2 composantes devraient également présenter des compositions chimiques différentes. Dans la partie suivante, nous associons la variation de la teneur en azote de la plante au cours de la croissance à la variation du rapport de ces composantes.

\section{B. Relation entre teneur en azote et vitesse de crois- sance relative}

Nous désignons par $n_{1}$ et $n_{2}$ les teneurs en azote de $M_{1}$ et $M_{2} ; n_{1}$ et $n_{2}$ sont supposées constantes pour une condition d'environnement donnée. Le contenu en azote de la plante au temps $t$ est donné par

$$
N(t)=n_{1} M_{1}(t)+n_{2} M_{2}(t) \text {. }
$$

La teneur en azote de la plante s'exprime alors sous la forme

$$
n(t)=\frac{N(t)}{M(t)}=\left(n_{1}-n_{2}\right) p_{1}(t)+n_{2} .
$$


TABLEAU 1

Valeur des coefficients des équations (3) et (9). Le coefficient $k_{1}$ de l'équation (3) $\frac{d M}{d t}=k_{1} M_{1}$ a été calculé à partir de la pente initiale des courbes de croissance expérimentales. Les coefficients a et b de l'équation $(9) n(t)=a r(t)+b$ ont été déterminés à partir d'une régression linéaire ; $n_{1}$ et $n_{2}$ sont déduits de $a$ et $b$ à l'aide des relations (10) $a=\frac{n_{1}-n_{2}}{k_{1}}$ et $b=n_{2}$.

Value of the coefficients of the equations (3) and (9). The $k_{l}$ coefficient of equation (3) $\frac{d M}{d t}=k_{1} M_{1}$ was calculated from the initial slope of experimental growth curves. The a and $b$ coefficients of equation (9) $n(t)=$ ar $(t)+b$ were determined from a linear regression analysis ; $n_{1}$ and $n_{2}$ were deduced from $a$ and $b$ using relationships (10) $a=\frac{n_{1}-n_{2}}{k_{1}}$ and $b=n_{2}$.

\begin{tabular}{cccccccc}
\hline \hline $\begin{array}{c}\text { Température } \\
\left({ }^{\circ} \mathrm{C}\right)\end{array}$ & $\begin{array}{c}\text { Eclairement } \\
\left(\mathrm{W} . \mathrm{m}^{-2}\right)\end{array}$ & $\begin{array}{c}\text { Concentration } \\
\text { en nitrate }(\mathrm{mM})\end{array}$ & $\begin{array}{c}\mathrm{k}_{1} \\
\left(\text { semaines }^{-1}\right)\end{array}$ & $\begin{array}{c}\mathrm{a} \\
\text { (semaines) }\end{array}$ & $\begin{array}{c}\mathrm{b}=\mathrm{n}_{2} \\
\left(\mathrm{~g}_{\mathrm{N}} / \mathrm{g}_{\mathrm{M}}\right)\end{array}$ & $\begin{array}{c}\mathrm{n}_{1}-\mathrm{n}_{2} \\
\left(\mathrm{~g}_{\mathrm{N}} / \mathrm{g}_{\mathrm{M}}\right)\end{array}$ & $\begin{array}{c}\mathrm{n}_{1} \\
\left(\mathrm{~g}_{\mathrm{N}} / \mathrm{g}_{\mathrm{M}}\right)\end{array}$ \\
\hline \multirow{2}{*}{10} & 55 & 0,5 & 0,72 & 0,038 & 0,015 & 0,027 & 0,042 \\
& 55 & 20 & 0,70 & 0,033 & 0,020 & 0,022 & 0,042 \\
25 & 35 & 0,5 & 1,85 & 0,016 & 0,018 & 0,030 & 0,048 \\
& 55 & 0,5 & 2,05 & 0,014 & 0,016 & 0,029 & 0,045 \\
& 85 & 0,5 & 2,25 & 0,013 & 0,016 & 0,029 & 0,045 \\
& 35 & 20 & 1,60 & 0,015 & 0,022 & 0,024 & 0,046 \\
& 55 & 20 & 1,75 & 0,014 & 0,022 & 0,025 & 0,047 \\
\hline \hline
\end{tabular}
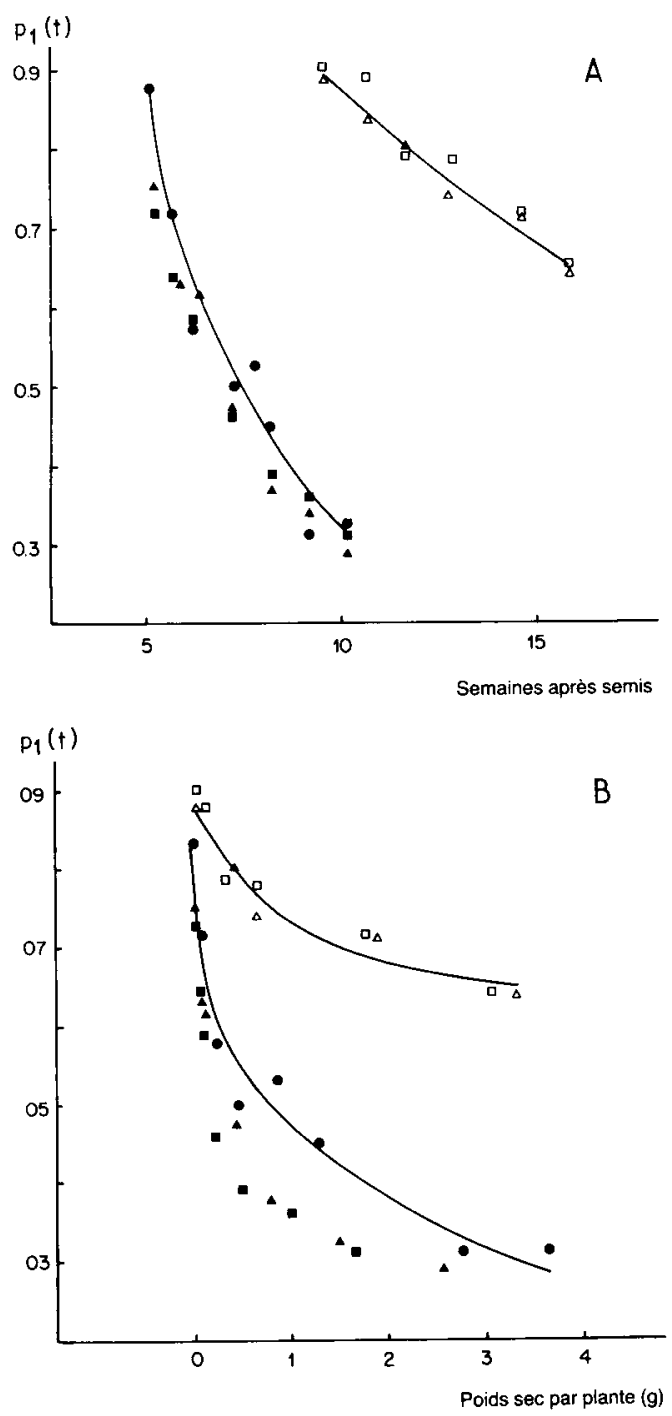

Figure 4

Variation de $p_{1}(t)$ en fonction du temps $(A)$ et en fonction du poids sec des plantes individuelles (B). Mêmes conventions que pour la figure 1 .

Dependence of $p_{I}(t)$ on $A$, time and $B$, dry weight of individual plants. Same conventions as in figure 1.
La vitesse de croissance relative (équation 6) et la teneur en azote de la plante (équation 8) sont proportionnelles à $p_{1}(t)$. En combinant ces 2 équations, on définit une relation linéaire entre la teneur en azote et la vitesse de croissance relative

$$
\begin{aligned}
& \mathrm{n}(\mathrm{t})=\operatorname{ar}(\mathrm{t})+\mathrm{b}, \\
& \mathrm{a}=\frac{\mathrm{n}_{1}-\mathrm{n}_{2}}{\mathrm{k}_{1}} \quad \text { et } \quad \mathrm{b}=\mathrm{n}_{2} .
\end{aligned}
$$

Le tableau 1 donne pour l'ensemble des conditions expérimentales la valeur des coefficients a et $b$ déterminée à l'aide d'une régression linéaire, ainsi que les valeurs de $n_{1}$ et $n_{2}$ calculées à partir des relations (10). La figure 5 montre l'accord entre les variations théoriques et expérimentales pour les traitements correspondant à la même concentration en nitrate du milieu nutritif (20 mM). Compte tenu de l'incertitude expérimentale sur $r(t)$, de l'ordre de 15 p. 100, et de l'imprécision sur la détermination de $\mathrm{n}_{2}$ à basse température (voir fig. 5), il est possible de déduire de cette analyse que la teneur en azote des 2 composantes est peu dépendante des conditions de culture. Les différences observées entre 10 et $25^{\circ} \mathrm{C}$ dans les variations de $\mathrm{n}(\mathrm{t})$ reflètent donc essentiellement les variations de $\mathrm{p}_{1}(\mathrm{t})$.

MIYASAKA et al. (1975) ont proposé pour le riz une relation linéaire entre la teneur en azote de la partie aérienne $\mathrm{n}_{\mathrm{a}}(\mathrm{t})$ et la vitesse de croissance relative de la surface foliaire $s_{a}(t)$, décrite par

$$
\mathrm{n}_{\mathrm{a}}(\mathrm{t})=\mathrm{a}_{1} \mathrm{~s}_{\mathrm{a}}(\mathrm{t})+\mathrm{b}_{1} \text {. }
$$

Cette relation se présente sous une forme très analogue à la relation (9), bien qu'il n'y ait pas rigoureusement correspondance entre les grandeurs mesurées et les variables du modèle. Ce résultat s'explique sans doute par le fait que l'azote de la plante se trouve essentiellement dans la partie aérienne et qu'il devrait exister une forte corrélation entre $s_{a}(t)$ et $r(t)$. Il est également intéressant d'observer que ces auteurs déterminent une valeur de $b_{1}$ comprise entre 1 et 2 p. 100 voisine de la valeur de $n_{2}$ calculée pour le 


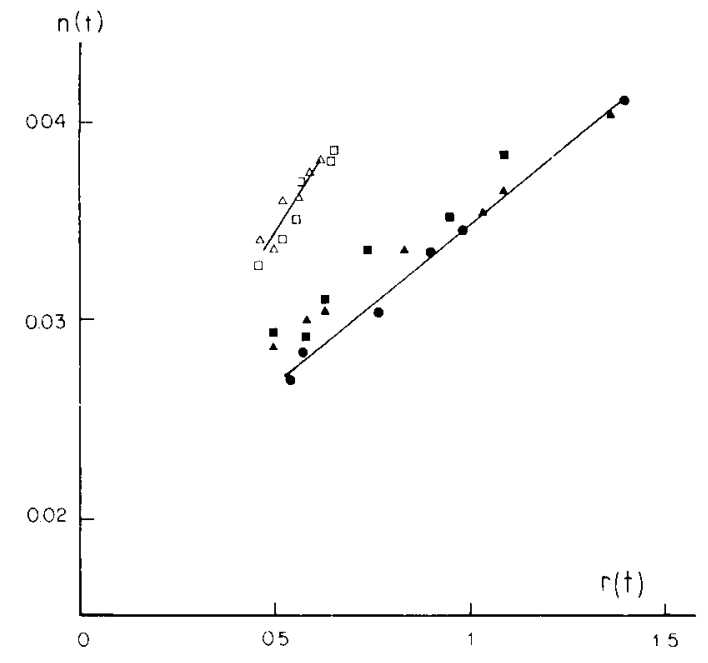

Figure 5

Relation entre la teneur en azote $n(t)$ et la vitesse de croissance relative $r(t)$ pour les plantes individuelles. Les courbes en trait continu, calculées à partir de l'équation (9), correspondent aux traitements de même concentration en nitrate du milieu nutritif (20 mM). Mêmes symboles que pour la figure 1 .

Relationship between nitrogen content, $n(t)$, and relative growth rate, $r(t)$, for individual plants. Continuous lines were calculated by equation (9) for the same condition of concentration of nitrate in the nutrient solution. Same symbols as in figure 1.

dactyle (tabl. 1). Nous rappelons que dans l'approche proposée, cette valeur mesure la teneur en azote de la composante $\mathrm{M}_{2}$ non associée aux processus de production de biomasse.

\section{Relation entre teneur en azote et biomasse}

La précédente description établit une relation entre la teneur en azote de la plante et la vitesse de croissance relative. Il est possible d'obtenir une relation entre la teneur en azote et le poids sec $\mathbf{M}$ de la plante en exprimant $r$ à l'aide d'une fonction de croissance usuelle (RICHARDS, 1969 ; HUNT, 1982) décrivant empiriquement, mais avec une bonne précision, la variation de $\mathbf{M}$ au cours du temps. Nous discutons brièvement certaines fonctions de croissance, de façon à justifier la fonction que nous utilisons.

La fonction de croissance, la plus couramment citée, est la fonction logistique décrite par l'équation

$$
\frac{\mathrm{d} M}{\mathrm{dt}}=\mathrm{C}_{1} \mathrm{M}-\mathrm{C}_{2} \mathrm{M}^{2} \text {. }
$$

Nous avons précédemment montré que cette fonction ne permettait pas une description satisfaisante des courbes expérimentales de croissance aux grandes valeurs de biomasse (CALOIN \& YU, 1982). Sur la base des résultats que nous présentons, il est également possible d'arriver à cette conclusion en observant que la vitesse de croissance relative,

$$
\mathrm{r}=\frac{1}{\mathrm{M}} \frac{\mathrm{dM}}{\mathrm{dt}}=\mathrm{C}_{1}-\mathrm{C}_{2} \mathrm{M}
$$

devrait décroître linéairement avec $\mathrm{M}$. Ce comportement n'est pas en accord avec les données de la figure
$2 \mathrm{~B}$, où l'on note que $\mathrm{r}(\mathrm{t})$ présente une concavité positive, très accentuée à $25^{\circ} \mathrm{C}$.

La fonction de GOMPERTZ ou la fonction puissance conduisent à une meilleure description des cinétiques expérimentales. Dans ce développement, nous utilisons la fonction puissance qui conduit à des expressions analytiques plus simples. Cette fonction, proposée par GREGORY (1928) peut être définie par l'équation

$$
\frac{\mathrm{dM}}{\mathrm{dt}}=\mathrm{kM}^{\alpha} \quad \text { avec } \quad 0<\alpha \leqslant 1,
$$

$\mathrm{k}$ et $\alpha$ sont des constantes pour un environnement donné. La vitesse de croissance relative est donnée par

$$
\mathrm{r}=\mathrm{kM}^{\alpha-1} \text {. }
$$

Dans le cas $\alpha=1$, la vitesse de croissance relative est constante et la croissance est exponentielle. On peut vérifier que cette expression de $r$ s'accorde avec les données de la figure $2 \mathrm{~B}$ (fonction décroissante en fonction de $M$, concavité positive).

En tenant compte de cette expression, l'équation (9) devient

$$
\mathrm{n}=\mathrm{AM}^{\alpha-1}+\mathrm{B}
$$$$
\text { avec } \mathrm{A}=\mathrm{ak} \text { et } \mathrm{B}=\mathrm{b} \text {. }
$$

Nous avons contrôlé la validité de cette relation à l'aide des données de la figure 3B. La valeur des coefficients $\mathrm{A}, \mathrm{B}$ et $\alpha$ a été déterminée à l'aide d'une procédure de régression non linéaire (RALSTON, 1981). Les résultats de cette analyse sont portés dans le tableau 2. Les courbes en trait continu de la figure $3 B$ montrent, aux 2 températures, l'accord avec les traitements correspondant à la même concentration en nitrate du milieu nutritif $(20 \mathrm{mM})$. On note que le coefficient B obtenu dans cette analyse n'est pas très différent du coefficient $b$ du tableau 1 , ce qui justifie également le choix de la fonction de croissance utilisée.

L'équation (16) définit ainsi une relation entre la diminution de la teneur en azote de la plante au cours de la croissance et le comportement cinétique. Dans le cas de la croissance exponentielle $(\alpha=1)$, la teneur en azote est constante. La variation de la teneur en azote devrait être d'autant plus accentuée que $\alpha$ est plus proche de 0 . Pour illustrer ce résultat, nous avons comparé les valeurs de $\alpha$ déterminées dans cette analyse avec celles directement obtenues en analysant les courbes expérimentales de croissance à partir de la relation (14) (CALOIN \& YU, 1982, CALOIN et al., 1983). Ces valeurs de $\alpha$, notées $\alpha_{c}$, sont portées dans lè tableau 2. On observe un bon accord entre ces 2 déterminations de $\alpha$. On en déduit que la relation (16), bien qu'empirique, permet d'établir une correspondance simple entre teneur en azote et cinétique de croissance par l'intermédiaire du coefficient $\alpha$.

Il est intéressant de rapprocher cette relation de la loi de dilution proposée par SALETTE \& LEMAIRE (1981) qui s'exprime sous la forme

$$
\mathrm{n}_{\mathrm{s}}=\mathrm{pM}_{\mathrm{s}}^{-\mathrm{q}}
$$

où $\mathrm{n}_{\mathrm{s}}$ est la teneur en azote de la matière sèche récoltable $\mathrm{M}_{\mathrm{s}}$; $\mathrm{p}$ et $\mathrm{q}$ sont des constantes pour un environnement donné. Cette relation a été vérifiée pour le dactyle (cv. «Lucifer ») et la fétuque élevée (cv. 
TABLEAU 2

Analyse de la variation de la teneur en azote des plantes individuelles au cours de la croissance. Valeur des coefficients $A, B$ et $\propto$ de l'équation (I6) $n(t)=A M^{\alpha-1}+B$. La valeur du coefficient $\propto$ est comparée avec celle obtenue en analysant les courbes de croissance expérimentales à partir de l'équation (14) $\frac{d M}{d t}=k M^{x}$. Cette valeur de $\alpha$ est notée $\alpha_{c}$. Ces déterminations ont été effectuées à l'aide d'une procédure de régression non linéaire. Analysis of the variation of nitrogen content of individual plants during growth. Value of the $A, B$ and $\alpha$ coefficients of equation (16) $n(t)=A M^{\alpha-1}+B$. The value of the $\alpha$ coefficient is compared with that obtained in the analysis of experimental growth curves using equation (14) $\frac{d M}{d t}=k M^{\alpha}$. This $\alpha$ value is denoted $\alpha_{c}$. These determinations were made using a non linear regression procedure.

\begin{tabular}{|c|c|c|c|c|c|c|}
\hline $\begin{array}{c}\text { Température } \\
\left({ }^{\circ} \mathrm{C}\right)\end{array}$ & $\begin{array}{c}\text { Eclairement } \\
\left(\mathrm{W} . \mathrm{m}^{-2}\right)\end{array}$ & $\begin{array}{c}\text { Concentration } \\
\text { en nitrate }(\mathrm{mM})\end{array}$ & $\begin{array}{c}\mathrm{A} \\
\left(g^{1-\alpha}\right)\end{array}$ & $\begin{array}{c}B \\
\left(g_{N} / g_{M}\right)\end{array}$ & $\alpha$ & $\alpha_{\mathrm{c}}$ \\
\hline \multirow[t]{2}{*}{10} & 55 & 0,5 & 0,016 & 0,018 & 0,89 & 0,94 \\
\hline & 55 & 20 & 0,015 & 0,023 & 0,90 & 0,92 \\
\hline \multirow[t]{6}{*}{25} & 35 & 0,5 & 0,012 & 0,016 & 0,74 & 0,76 \\
\hline & 55 & 0,5 & 0,010 & 0,018 & 0,78 & 0,78 \\
\hline & 85 & 0,5 & 0,010 & 0,014 & 0,77 & 0,79 \\
\hline & 35 & 20 & 0,009 & 0,021 & 0,74 & 0,77 \\
\hline & 55 & 20 & 0,011 & 0,018 & 0,76 & 0,73 \\
\hline & 85 & 20 & 0,013 & 0,019 & 0,73 & 0,76 \\
\hline
\end{tabular}

« Ludelle ») pendant plusieurs cycles de culture en milieu naturel. La validité des relations (16) et (18) impliquerait essentiellement une proportionnalité entre matière sèche récoltée et poids total de la matière sèche. Il est à noter également que les courbes de variation de $n_{s}$ en fonction de $M_{s}$ présentées par ces auteurs ne sont pas incompatibles avec l'existence d'un terme résiduel $b$ relatif à la teneur en azote de la matière sèche en fin de croissance.

En conclusion, l'analyse des cinétiques de croissance végétative du dactyle en environnement contrôlé nous a conduits à proposer un modèle de croissance à 2 composantes différenciées selon leur fonction dans les mécanismes de production de biomasse. Le modèle permet de rendre compte de la variation de la teneur en azote de la plante au cours de la croissance dans des conditions expérimentales très variées et relie cette variation au comportement cinétique. Cette approche demande cependant à être développée de façon à introduire la répartition des assimilats entre les parties aérienne et racinaire.

Reçu le 10 janvier 1985 Accepté le ler octobre 1985.

\section{REMERCIEMENTS}

Nous remercions Monsieur le Professeur M. Gounot pour les fructueuses discussions que nous avons eues avec lui et la critique du manuscrit.

Nous remercions également la Station d'Agronomie de l'I.N.R.A. à Colmar qui nous a accueillis dans ses laboratoires pour le dosage de l'azote des échantillons.

\section{ANNEXE}

Composition des milieux nutritifs.

Composition of the nutrient solutions.

Solution nutritive $0,5 \mathrm{mM} \mathrm{NO}_{3}^{-}$.

Nutrient solution $0.5 \mathrm{mM} \mathrm{NO}_{3}^{-}$.

\begin{tabular}{lccccc}
\hline meq/l & $\mathrm{NO}_{3}^{-}$ & $\mathrm{PO}_{4}^{3-}$ & $\mathrm{SO}_{4}^{2-}$ & $\mathrm{Cl}^{-}$ & Total \\
\hline $\mathrm{K}^{+}$ & & 1,47 & 1,73 & & 3,20 \\
\hline $\mathrm{Ca}^{++}$ & 0,5 & & & 5,34 & 5,84 \\
\hline $\mathrm{Mg}^{++}$ & & & 2,32 & & 2,32 \\
\hline $\mathrm{H}^{+}$ & & 2,94 & & & 2,94 \\
\hline Total & 0,5 & 4,41 & 4,05 & 5,34 & 14,30 \\
\hline \hline
\end{tabular}

Solution nutritive $20 \mathrm{mM} \mathrm{NO}_{3}^{-}$.

Nutrient solution $20 \mathrm{mM} \mathrm{NO}_{3}^{-}$.

\begin{tabular}{lccccc}
\hline \hline meq/l & $\mathrm{NO}_{3}^{-}$ & $\mathrm{PO}_{4}^{3-}$ & $\mathrm{SO}_{4}^{2-}$ & $\mathrm{Cl}^{-}$ & Total \\
\hline $\mathrm{K}^{+}$ & 5,65 & 1,47 & & 0,1 & 7,22 \\
\hline $\mathrm{Ca}^{++}$ & 13,18 & & & & 13,18 \\
\hline $\mathrm{Mg}^{++}$ & 1,17 & & 4,05 & & 5,22 \\
\hline $\mathrm{H}^{+}$ & & 2,94 & & & 2,94 \\
\hline Total & 20 & 4,41 & 4,05 & 0,1 & 28,56 \\
\hline \hline
\end{tabular}

Micro-éléments communs aux deux solutions nutritives $(\mathrm{mg} / \mathrm{l})$ :

Micro-nutrients for both nutrient solutions (mg/l) :

B : 0,52: Cu : 0,0305; Fe : 8,835; Mn : 0,55; Mo : 0,0217; Zn : 0,0682 


\section{RÉFÉRENCES BIBLIOGRAPHIQUES}

Blondon F., 1964. Contribution à l'étude du développement des graminées fourragères ray-grass et dactyle. Thèse Doct. Etat, Fac. Sci., Paris, 131 p.

Caloin M., Yu O., 1982. An extension of the logistic model of plant growth. Ann. Bot., 49, 599-607.

Caloin M., Yu O., 1984. Analysis of the time course of change in nitrogen content in Dactylis glomerata L. using a model of plant growth. Ann. Bot., 54, 69-76.

Caloin M., Malonga A., Tricart E., 1983. Analyse de la cinétique de croissance du Dactylis glomerata L. par une approche morphogénétique. Oecol. Flant., 4 (18), 103-116.

Champagnat P., Ozenda P., Baillaud L., 1969. Croissance, Morphogenèse, Reproduction. Biologie Végétale, vol. III, Masson Paris, $510 \mathrm{p}$.

Clement C. R., Hopper M. J., Jones L. H. P., 1978. The uptake of nitrate by Lolium perenne from flowing nutrient solution. $J$. exp. Bot., 25, 453-464.

Coïc Y., Lesaint C., Cholet Y., 1974. Accumulation comparée du cuivre dans les racines et-parties aériennes de plantes fourragères en fonction de l'alimentation en cuivre et de la nature nitrique ou nitrico-ammoniacale de l'alimentation azotée. C. $R$. Acad. Agric. Fr., 60 (15), 1162-1171.

Cooper A. J., Thornley J. H. M., 1976. Response of dry matter partitioning, growth, and carbon and nitrogen levels in the tomato plant to changes in root temperature : experiment and theory. $A n n$. Bot., 40, 1139-1152.

Evans G. C., 1972. The Quantitative Analysis of Plant Growth. Blackwell Sci. Publ., Oxford, 734 p.

Frissel M. J., Van Veen J. A., 1981. Simulation of Nitrogen Behaviour of Soil-Plant Systems. Pudoc, Wageningen, 277 p.

Gillet M., 1980. Les graminées fourragères. Gauthier-Villars, Paris, $306 \mathrm{p}$.

Gillet M., Lemaire G., Gosse G., 1984. Essai d'élaboration d'un schéma global de la croissance des graminées fourragères. Agronomie, 4 (1), 75-82.

Greenwood D. J., 1981. Crop Response to Agronomic Practice, 195-216. In D. A. Rose \& D. A. Charles-Edward : « Mathematics and Plant Physiology ». Academic Press, London, 320 p.

Gregory F. G., 1928. Studies in energy relations of plants. II. The effect of temperature on increase in area of leaf surface and in dry weight of Cucumis sativus. Part 1. The effect of temperature on the increase in area of leaf surface. Ann. Bot. (Old Series), 42, 462-507.

Heller R., 1969. Nutrition et Métabolisme. Biologie Végétale, vol. II, Masson, Paris, 578 p.

Hunt R., 1982. Plant Growth Curves. Edward Arnold, 248 p.

Lemaire G., Salette J., 1984. Relation entre dynamique de croissance et dynamique de prélèvement d'azote pour un peuplement de graminées fourragères. I. - Etude de l'effet du milieu. Agronomie, $4(5), 423-430$

Miyasaka A., Murata Y., Iwata T., 1975. Leaf area development and leaf senescence in relation to climatic and other factors, 72-85. In Y. MURATA : "Crop productivity and solar energy utilization in various climates in Japan ". Univ. of Tokyo Press, Tokyo, 244 p.

Ralston M., 1981. Derivative free non linear regression, 484-489. In W. J. Dixon \& M. B. Brown : " BMDP Biomedical Computer Programs ». Univ. of Calif. Press, Los Angeles, 880 p.

Richards F. J., 1969. The quantitative analysis of growth, 1-76. In F. C. STEWARD : "Analysis of Growth: Behaviour of Plants and their Organs. Plant Physiology. A treatise ». Vol. VA. Academic Press, London, 435 p.

Salette J., Lemaire G., 1981. Sur la variation de la teneur en azote des graminées fourragères pendant leur croissance : formulation d'une loi de dilution. C. R. Acad. Sci. Paris, 292, 875-878.

Warren Wilson J., 1972. Control of crop processes, 7-30. In A. R Rees, K. E. Cockshull, D. W. Hand \& R. G. Hurd : “ Crop Pro cesses in Controlled Environnements ". Academic Press, London, $391 \mathrm{p}$. 